\title{
Molecular cloud shredding in the Galactic Bar
}

\author{
H. S. Liszt
}

National Radio Astronomy Observatory, 520 Edgemont Road, 22903-2475 Charlottesville, VA, USA

e-mail: hliszt@nrao.edu

Received 18 August 2005 / Accepted 13 October 2005

\begin{abstract}
Seen just outside the innermost regions of the galactic center, the kinematics of molecular gas are dominated by a handful of compact but unusually broad-lined features of enigmatic origin. We show, using previous data, that there is a family of such features whose members are distinguished morphologically by their extreme vertical extension, perpendicular to the inclined plane of the overall gas tilt. Having isolated the features spatially, we mapped them with varying degrees of completeness at high resolution $\left(1^{\prime}\right)$ in lines of ${ }^{12} \mathrm{CO},{ }^{13} \mathrm{CO}$ and $\mathrm{CS}$. Although very broad profiles exist in some individual beams, more generally we resolved the kinematics into spatial gradients which earlier were smeared in broader beams to form wider lines. The largest apparent velocity gradients are typically with respect to galactic latitude but motions are confined to the range of velocities inside the galactic terminal velocity, indicating that it is the galactic gravitational potential which is being tapped to create the observed kinematics.

We interpret the broad-lined features qualitatively in terms of recent hydrodynamical models of gas flow in strongly barred galaxies: standing shocks which occur where gas enters the Galactic dust lane can account for the presence of broad lines over small spatial volumes wherever molecular gas is actually engaged in this process. To interpret the dynamical sequencing of the complex behaviour seen within the broad-line features we discuss how the Sun must be oriented with respect to the bar. In doing so, we identify the nuclear star-forming rings seen in other galaxies with the complex of giant H II regions Sgr A, B, C etc. and show that the kinematics are as expected for a ring of radius $175 \mathrm{pc}$ (for a Sun-center distance of $8.5 \mathrm{kpc}$ ) rotating at about $210 \mathrm{~km} \mathrm{~s}^{-1}$. Gas having clear and strong outward-directed non-circular motion around $l=0^{\circ}$ (the famous "expanding molecular ring") is then associated with the "spray" of incoming gas at the inner ends of the dust lane, defining a more nearly end-on viewing angle for the bar.

Using the inferred geometry, we construct a narrative for the behaviour of the feature most completely mapped here, at $l=5.4^{\circ}$, whereby gas basically falls out of the sky and is concentrated into the observed dense, bright molecular core before being shredded and sucked into the inflow of the dust lane $100 \mathrm{pc}$ or more below the nominal galactic equator. From there it is recycled and lifted back into the more nearly equatorial region of the nuclear ring. Of course, the vertical structure of this and the other features, and the overall tilt of the dust lane and inner-galaxy gas layer, all remain to be discussed theoretically.
\end{abstract}

Key words. Galaxy: nucleus - ISM: clouds - ISM: molecules

\section{Introduction}

It seems generally agreed that the large-scale neutral gas motions observed in the center of the Milky Way (Cohen \& Davies 1976; Bania 1977; Burton \& Liszt 1978; Liszt \& Burton 1978, 1980; Rohlfs \& Braunsfurth 1982; Burton \& Liszt 1983; Bitran et al. 1997) result from the presence of a substantial bar. Kinematic and dynamical bar models which have been tailored to fit the galactic gas observations (Peters 1975; Liszt \& Burton 1980; Rohlfs 1983; Mulder \& Liem 1986; Binney et al. 1991; Jenkins \& Binney 1994; Gerhard 1996; Weiner \& Sellwood 1999; Fux 1999) have some (not all) important common elements. The near end is found to occur in the first quadrant of galactic longitude and the more recent gas-dynamical models are "fast", reaching corotation at galactocentric distances of a few kpc. In stellar dynamical models, elongated $x_{1}$ orbits comprise the larger body of the bar while smaller, more nearly circular $x_{2}$ orbits occur nearer the center (Contopoulos \& Grosbol 1989; Athanassoula 1992): these have analogues in the gas motions (Binney et al. 1991). Differences among the models occur in the angle between the bar major axis and our line of sight to the galactic center (ranging from $15^{\circ}-45^{\circ}$ ) and the radius of corotation, depending on what evidence is accorded the greatest importance.

It is a truism that phenomena viewed relatively nearby in the Galaxy are often greatly elucidated when seen from outside in other galaxies and it has recently become possible to observe the gasdynamical effects of strong bars in other galaxies in greater detail. Figure 1, from Regan et al. (1999) shows schematically the gas motions in the hydrodynamical model of gas flow in a strong extragalactic bar; two prospective viewing geometries are noted, the differences between which will be discussed later in this work (Sect. 4). The arrow-annotated ridge lines in Fig. 1 are (in the rotation frame) standing shocks, 


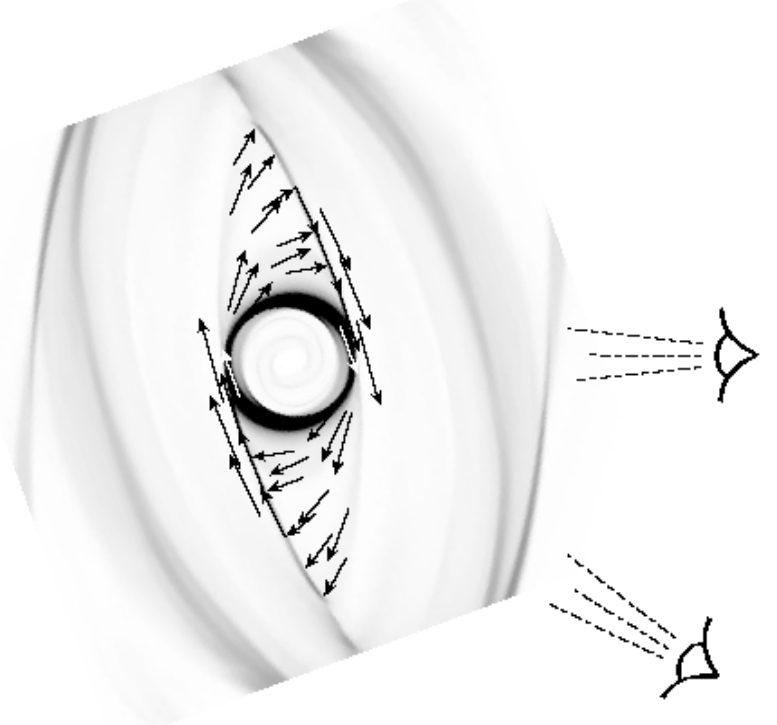

Fig. 1. Possible geometries for viewing the inner-Galaxy bar, after Fig. 9 of Regan et al. (1999). The velocity vectors shown are in the rotating frame of the bar.

which, in the $x_{1}$ region, are seen optically as dust lanes; these are straighter in stronger bars. In this model the interface between the regions of dominance of the stellar $x_{2}$ and $x_{1}$ orbit types produces a violent spray of material which has flowed inward along the length of the dust lane shocks, and (in the case shown) a non-stationary nuclear ring over which intense star formation is observed in many barred external galaxies (Regan $\&$ Teuben 2003, 2004). Motions in the star-forming nuclear ring are predominantly circular (i.e. $x_{2}$-like). The alignment of the dust lines and the presence of the spray and nuclear starforming ring are all contingent on the bar properties.

These phenomena are present in other galaxies; are they also present in our own galaxy? In the Milky Way the nuclear star-forming ring should probably be identified with the continuum sources Sgr A-E, but any such match remains to be assessed quantitatively (however, see Stark et al. 2004): here (Sect. 4) we show that the kinematics of the inner-galaxy HII regions in fact fit the expected pattern and define fairly well a ring of radius $\approx 70^{\prime}=175 \mathrm{pc}$. Additionally, we associate the region of spray in the Fig. 1 model with the so-called "expanding molecular ring" (EMR) gas in the galactic center, and the presence of strong-noncircular motions in observations near $l=0^{\circ}$ determines which of the possible viewing schema (more nearly along or across the bar) pertains to our situation within the Milky Way: this association between the EMR and gas spray has also not been made previously.

Conversely, some important aspects of the galactic center gas distribution remain to be explained in the context of bar models. One of these (which is quite prominent in the gas discussed here) is a large-scale tilt of the gas away from the galactic equator (Cohen \& Davies 1976; Burton \& Liszt 1978; Liszt \& Burton 1978, 1980). The early hope that such a geometry could be a stable feature of a triaxial Milky Way bulge (Lake \& Norman 1983) seems not to have been realized

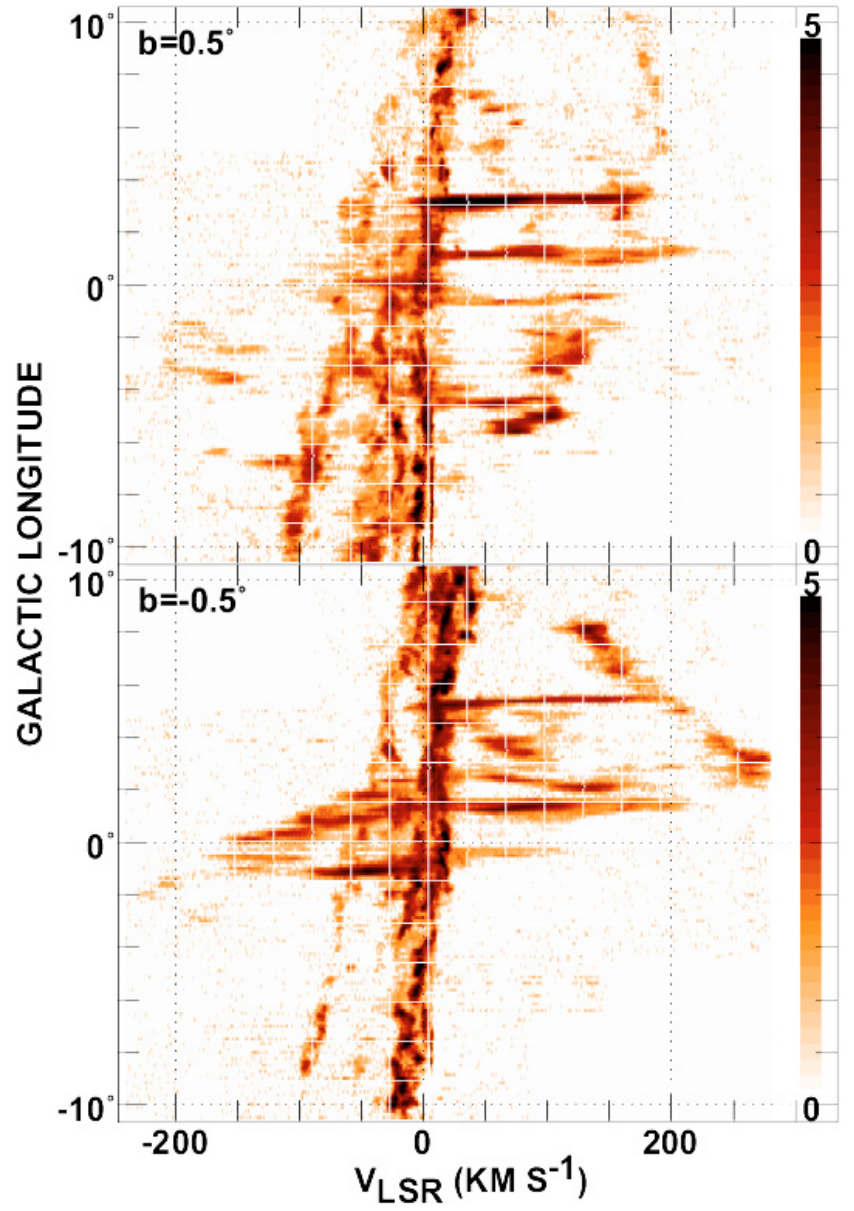

Fig. 2. Longitude-velocity diagrams for $\lambda 2.6 \mathrm{~mm}{ }^{12} \mathrm{CO}$ emission at $0.125^{\circ}$ spacing and $8^{\prime}$ resolution from the dataset of Bitran et al. (1997).

(Vietri 1986), although examples of mis-aligned inner gas disks in other galaxies are increasingly common. However, the point of the present work is to address another enigmatic aspect of the neutral gas distribution in the inner kpc of galactocentric radius. Away from the galactic equator, and/or outside the inner few hundred pc, the most prominent features are a small number of highly localized but extraordinarily wide lines which have no parallel in other studies of galactic structure. Examples of the broad-line phenomenon as seen in the CO survey of Bitran et al. (1997) are shown in Fig. 2; see Sect. 2 for a full discussion of this data. Perusal of just these two cuts above and below the plane shows that there are nominally similar features at perhaps a half-dozen longitudes. The appearance of the broad-lined features is perhaps even more striking in species like $\mathrm{OH}$ (Boyce et al. 1989), $\mathrm{H}_{2} \mathrm{CO}$ (Zylka et al. 1992) or HCN (Lee 1996), which, at $l>2^{\circ}$, do not show the zero-velocity and/or terminal velocity ridges.

The issues for interpretation of the broad-lined galactic features have centered on the source of the apparently enormous kinetic energies implied by the large line widths. If the high masses typically found for GMC's (say, $10^{5}-10^{6} M_{0}$ ) and the large linewidths are used to form a kinetic energy with a characteristic velocity of order $100 \mathrm{~km} \mathrm{~s}^{-1}$, one finds $E \sim$ $10^{53}-10^{54} \mathrm{erg}$ (also see Boyce et al. 1989 and Thaddeus 1985). 
It has been hard to imagine how this prodigious amount of purely mechanical energy might be manifested within regions of the size of the wide-lined features as they appear on the sky (perhaps $30 \mathrm{pc}$ across in Fig. 2), or how the material would remain neutral, or stay so assiduously within the normal terminal velocity associated with seemingly undisturbed material. There is no continuum radiation or other manifestation of energetic activity for these features, as far as is known, beside the neutral gas kinematics.

Although the phenomenon was explained by Thaddeus (1985) (using the data in Fig. 2) in terms of explosive phenomena in the galactic center (perhaps following Oort 1977) or described simply as velocity dispersion by Kumar \& Riffert (1997), the preferred alternative has been to disperse the molecular gas into unrelated clouds which are coincidentally viewed over long paths down the elongated bar structure in the galactic center. The presence of dust lanes down $x_{1}$ orbits is cited, consistent with an orientation of the bar nearly along the line of sight to the galactic center (Stark \& Bania 1986). Stark \& Bania (1986) showed a higher-resolution integrated intensity CO map of the feature at $l=3.2^{\circ}$ (aka Clump 2 of Bania 1977); no kinematics were shown. In their description, galactic tidal forces strip material off some 16 dense and quite massive cloud cores (individually, $5 \times 10^{5} \mathrm{M}_{0}$ ) forming locally broader-lined, tenuous, extended CO-bearing envelopes whose profiles blend along the line of sight to form the observed, yet much wider, overall profiles. A nominally similar viewpoint was adopted by Boyce et al. (1989). Lee et al. (1999) described the feature at $l=5.4^{\circ}$ as consisting of the combination of a spiral arm, elliptical ring and admixture of high-velocity-dispersion clouds, and ascribed Clump 2 to all of these plus " $x_{2}$ family components of the central concentration".

Explanations which disperse gas along extended sight lines are troubled by the hypothesis of so many "fingers of God" pointed conveniently toward the Sun. Here, we follow the recent insight of Fux (1999) into another possibility for the genesis of Clump 2 and the feature at $l=5.4^{\circ}$, which again has an analogue in extragalactic studies although it may not be so striking there. Regarding Fig. 1, we see that the gas motion changes direction very abruptly along the entire length of the narrow standing dust lane shock running down the bar. When this near right-angle turn is seen from outside, highly localized gas abruptly (very compactly) traverses very nearly the entire velocity range which is possible in the model at that longitude, so that spatially compact but very wide-lined features might be seen wherever enough gas is actually entering the dust lane, over a wide range of longitude. This occurs without recourse to viewing angle, coincidental superposition or long "Fingers of God". The small volume filling factor of molecular gas would seem to be enough to ensure that such events are occasional and well-separated in space and/or time and the abruptness of the shock makes the presence of wide lines largely independent of orientation (though the details and interpretation would differ). It affords the exciting possibility that we might view, in great detail, the actual sequence of processes by which the galactic dust lanes are fed and material channeled toward the nucleus.

In this work, we show first that there is a larger family of l-compact, wide-lined features. Family members are identifiable morphologically because they are distinguished by extraordinary elongation across the galactic equator, typically exceeding $100 \mathrm{pc}$ in vertical extent, while remaining compact in longitude; the features are often not much wider in longitude than a single molecular cloud. Having identified the features in this way, we fully mapped the wide-lined feature centered near $l=5.4^{\circ}$ in ${ }^{12} \mathrm{CO}$ and strip-mapped along its length in ${ }^{13} \mathrm{CO}$ and $\mathrm{CS}(J=2-1)$. We also strip-mapped along the other features which could be identified, some five in all, in various mm-wave emission lines. In this way we show that both Clump 2 and the feature centered near $l=5.4^{\circ}$ have the same kinematic structure and must arise from the same phenomenon. Other features seen closer to the center show some aspects of the same internal kinematics, but have somewhat different large-scale behaviour.

The arrangement of this work is as follows. In Sect. 2 we describe the large-scale arrangement of molecular gas as seen in the low-resolution CO survey of Bitran et al. (1997), for the purpose of identifying the full range of wide-line behaviour. In Sect. 3 we describe the new observational material taken for the present purposes, and describe the results of mapping the various features in several mm-wave emission lines of CO and CS. In Sect. 4 we integrate the Milky Way observations with the current expectation for gas behaviour in strongly-barred systems, identifying the nuclear ring, spray region, and shocked dust lane gas.

\section{The large-scale behaviour of molecular gas in the Galactic Center}

Until very recently, the only comprehensive and truly largescale study of the molecular gas distribution outside the immediate vicinity of the galactic nucleus was that of Bitran et al. (1997), who mapped the ${ }^{12} \mathrm{CO}(J=1-0)$ emission distribution at $8^{\prime}$ resolution and $0.125^{\circ}$ beam spacing at $|b| \leq 1.0^{\circ}$, and more coarsely at larger $|b|$. Sawada et al. (2001) re-observed the inner $\pm 6^{\circ}$ of longitude in the $J=2-1$ line with somewhat better latitude coverage, but with no better beam spacing or resolution.

Figure 2 shows two longitude-velocity cuts made through the datacube of Bitran et al. (1997). The presence of several broad, strong lines extending between (roughly) $0 \mathrm{~km} \mathrm{~s}^{-1}$ and the terminal velocity is obvious. At some positions the terminal velocity is not actually occupied by emission, because of the tilt of the gas (see just below), but the wide-line kinematics nonetheless do not extend beyond the inherent terminal velocity at their longitude. Most of the broad lines appear at positive longitude, or at positive velocity. The broad line at negative longitude and velocity in the lower panel is part of the parallelogram pattern which Binney et al. (1991) used to demonstrate the $x_{1}-x_{2}$ orbit separation in H I and CO data.

Our attempts to trace the broad line features in such cuts over a range of latitude foundered under the burden of complexity of the observed phenomena. A more fruitful approach is shown in Fig. 3, where the gas is described in more nearly spatial terms. In the top panel, we show the distribution of the line profile integral (units of $\mathrm{K} \mathrm{km} \mathrm{s}^{-1}$ ) taken over the range $30<|v|<180 \mathrm{~km} \mathrm{~s}^{-1}$, which largely segregates emission inside the terminal velocity ridge over the range of displayed 


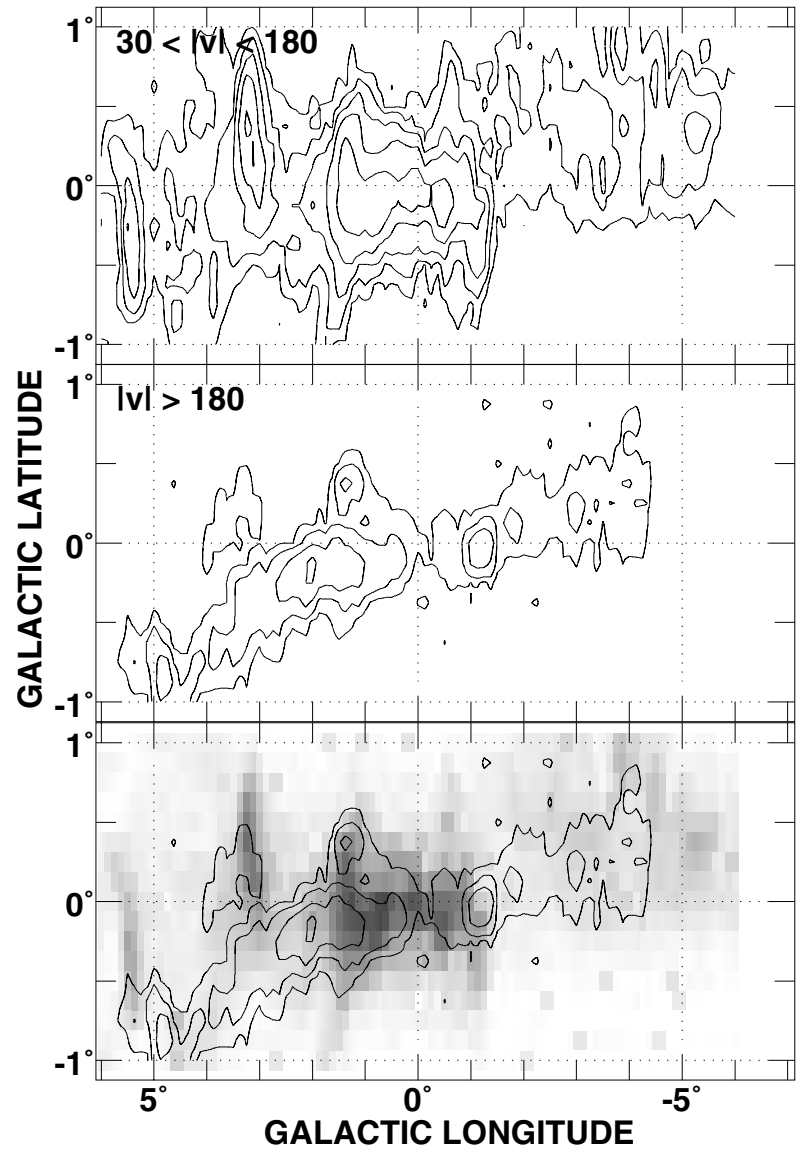

Fig. 3. Integrated intensity maps made from the ${ }^{12} \mathrm{CO}$ dataset of Bitran et al. (1997) at $8^{\prime}$ resolution. The panel at top includes galactic center gas below the terminal velocity; contour levels increase by factors of 2 from $100 \mathrm{~K} \mathrm{~km} \mathrm{~s}^{-1}$. The middle panel represents mainly the gas in the terminal velocity ridge, with contours increasing in steps of factors of 2 from $20 \mathrm{~K} \mathrm{~km} \mathrm{~s}^{-1}$. At bottom the two distributions are superposed. The vertical filaments at intermediate velocity at $l=-1.1^{\circ}$, $-0.5^{\circ}, 1^{\circ}-2^{\circ}, 3.1^{\circ}$ and $5.4^{\circ}$ all appear as uncharacteristically broad lines in Fig. 2.

longitude (somewhat more compact than in Fig. 2). The middle panel is for $|v| \geq 180 \mathrm{~km} \mathrm{~s}^{-1}$, which isolates the terminal velocity ridge. The bottom panel superposes these distributions, with the terminal-velocity ridge shown as contours and the interior gas as a gray-scale.

The tilt of the inner-galaxy molecular gas (Burton \& Liszt 1978; Liszt \& Burton 1980) is manifested in both velocity ranges in Fig. 3 but is especially clear in the terminal velocity ridge (compare with Fig. 3 in Liszt \& Burton 1980, for H I). That feature is always found well below the galactic equator at positive longitude and above it in the fourth longitude quadrant, in both $\mathrm{CO}$ and $\mathrm{H} \mathrm{I}$.

As is especially clear in the bottom panel, emission inside the terminal velocity is dominated by a small number of rather narrow, vertically-extended "streaks" and even the massive Sagittarius source complex makes little impression on the spatial scale of this diagram. Each of the vertical excrescenses is in fact identifiable as a broad-lined feature at some latitude. The wide-lined clouds at $l=5.4^{\circ}$ and $3.2^{\circ}$ are slightly but oppositely curved, and appear to terminate at the tilted terminalvelocity distribution. Closer to the center, where the tilt is less apparent, a feature between $l=+1^{\circ}$ and $+2^{\circ}$, with the stupendous vertical extent of $1.5^{\circ}$ or $225 \mathrm{pc}$, appears at both high positive and negative latitude, and also has a noticeable curvature where it is seen furthest below the galactic equator. One or two similar but weaker vertical extensions also exist at $-1^{\circ}<l<-0.5^{\circ}$, and the closer-in of these also appears to cross the plane substantially (this is shown in more detail below). A broad feature extending out to $v=+100 \mathrm{~km} \mathrm{~s}^{-1}$ at $l=-4.4^{\circ}, b=+0.5^{\circ}$ appears just North of gas forming Clump 1 of Bania (1977) and Bania et al. (1986). It may be present as a vertical extension in Fig. 3, but only weakly.

The two ranges covered in Fig. 3 really encompass just about all of the emission which can be associated with the galactic center, and it seems remarkable that so much of it can be decomposed into just these two components; the tilted terminal-velocity ridge (which in some viewing geometries should contain the bulk of any widely-distributed gas and in others is the dust-lane) and a few rather narrow, vertical excursions represented by the broad-lined features in Fig. 2.

\section{New observations of the wide line features}

\subsection{The data}

The observation that all of the wide-line features are so vertically extended informed nearly a week of on-the-fly (OTF)-mapping at the Steward Observatory $12 \mathrm{~m}$ telescope in 2002 May. In taking the data we position-switched to not toodistant reference positions chosen on the basis of the absence of emission from the inner Galaxy. Afterward, longer integrations were done on the off positions, switching against much more distant references, and these were added back into the data cubes to recapture emission from more local gas.

The per-beam on-source integration time was typically $10 \mathrm{~s}$ after gridding onto rectangular $15^{\prime \prime}$ pixels and the rms noise levels were typically $0.4 \mathrm{~K}$ in ${ }^{12} \mathrm{CO}(1-0), 0.09 \mathrm{~K}$ in ${ }^{13} \mathrm{CO}$ and $0.07 \mathrm{~K}$ in CS (2-1). The data were taken in $391 \mathrm{kHz}$ filters yielding resolution of $1.02 \mathrm{~km} \mathrm{~s}^{-1}, 1.06 \mathrm{~km} \mathrm{~s}^{-1}$, and $1.20 \mathrm{~km} \mathrm{~s}^{-1}$ in the ${ }^{12} \mathrm{CO},{ }^{13} \mathrm{CO}$, and $\mathrm{CS}$ lines, respectively. Velocities are measured with respect to the Local Standard of Rest and the brightness scale of data taken at the $12 \mathrm{~m}$ is the usual $T_{\mathrm{R}}^{*} \sim 0.7 T_{\mathrm{mb}}$. The spatial resolution of the $12 \mathrm{~m}$ telescope is approximately $1^{\prime}$ for the $\mathrm{CO}$ lines and $75^{\prime \prime}$ for CS. At the assumed galactic center distance of $8.5 \mathrm{kpc}, 1^{\prime}$ subtends $2.5 \mathrm{pc}$.

We mapped the region $5^{\circ}<l<6^{\circ},-1^{\circ}<b<0.5^{\circ}$ in the ${ }^{12} \mathrm{CO}(J=1-0)$ line and mapped a narrow vertical strip $\left(0.1^{\circ}\right.$ in width) in ${ }^{13} \mathrm{CO}$ and $\mathrm{CS}(J=2-1)$; data for $\mathrm{HCO}^{+}$were also taken but are not shown here. The integrated intensity map for ${ }^{12} \mathrm{CO}$ at $30<v<180 \mathrm{~km} \mathrm{~s}^{-1}$ and latitude-velocity diagrams at $l=5.4^{\circ}$ are all shown in Fig. 4 . The ${ }^{12} \mathrm{CO}$ mapping data for this feature are shown as moment maps over $15 \mathrm{~km} \mathrm{~s}^{-1}$-wide intervals in Fig. 5, as longitude-velocity diagrams in Fig. 6 and as latitude-velocity cuts in Fig. 7.

An integrated intensity map of ${ }^{12} \mathrm{CO}$ emission from Clump 2 was given previously by Stark \& Bania (1986). We strip-mapped a narrow swath along the vertical mid-line of Clump 2 in ${ }^{12} \mathrm{CO},{ }^{13} \mathrm{CO}$ and $\mathrm{CS}(J=2-1)$ and Fig. 8 shows latitude-velocity cuts at $l=3.15^{\circ}$ in these species. Comparison of Figs. 2 and 8 here with the earlier map suggests that the 


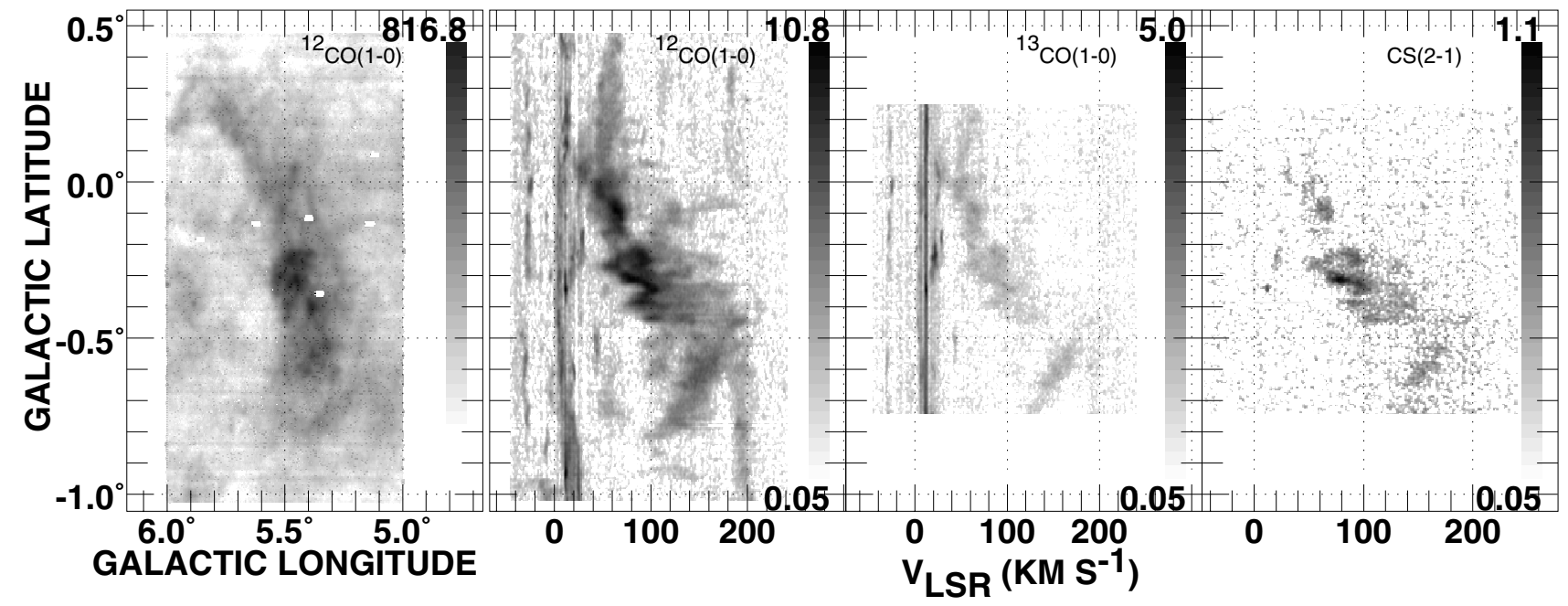

Fig. 4. An integrated intensity map of ${ }^{12} \mathrm{CO}$ emission over the range $30 \leq v \leq 180 \mathrm{~km} \mathrm{~s}^{-1}$ for the feature at $l=5.4^{\circ}$, and latitude-velocity cuts at $l=5.5^{\circ}$ for ${ }^{12} \mathrm{CO},{ }^{13} \mathrm{CO}$ and $\mathrm{CS}(J=2-1)$. Small blanked regions in the ${ }^{12} \mathrm{CO}$ image hide minor glitches during the observing and gridding.

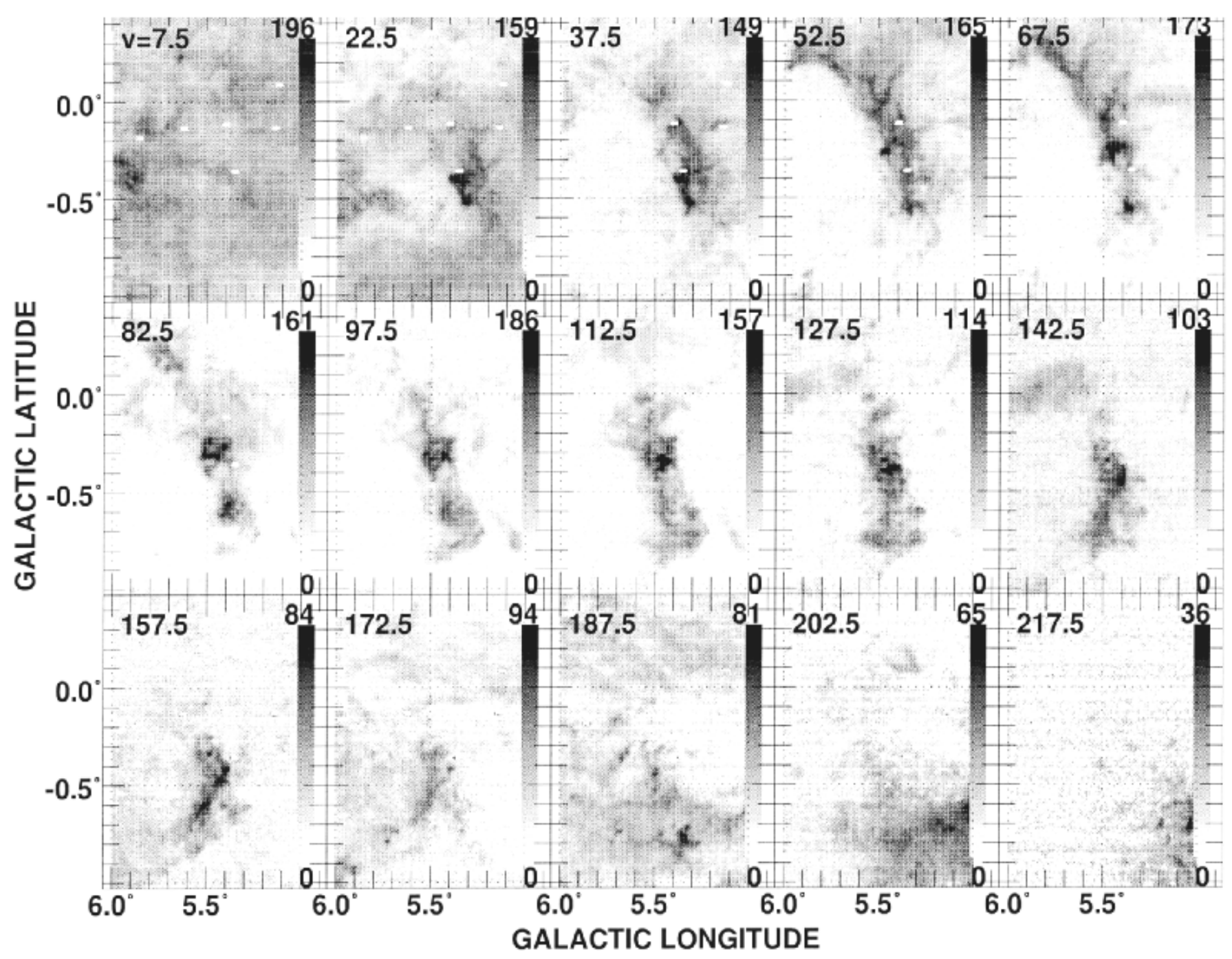

Fig. 5. Integrated-intensity maps (units of $\mathrm{K} \mathrm{km} \mathrm{s}^{-1}$ ) over $15 \mathrm{~km} \mathrm{~s}^{-1}$-wide velocity ranges, centered as indicated. The peak integrated intensity $\left(\mathrm{K} \mathrm{km} \mathrm{s}^{-1}\right)$ in each frame is shown at upper right. 


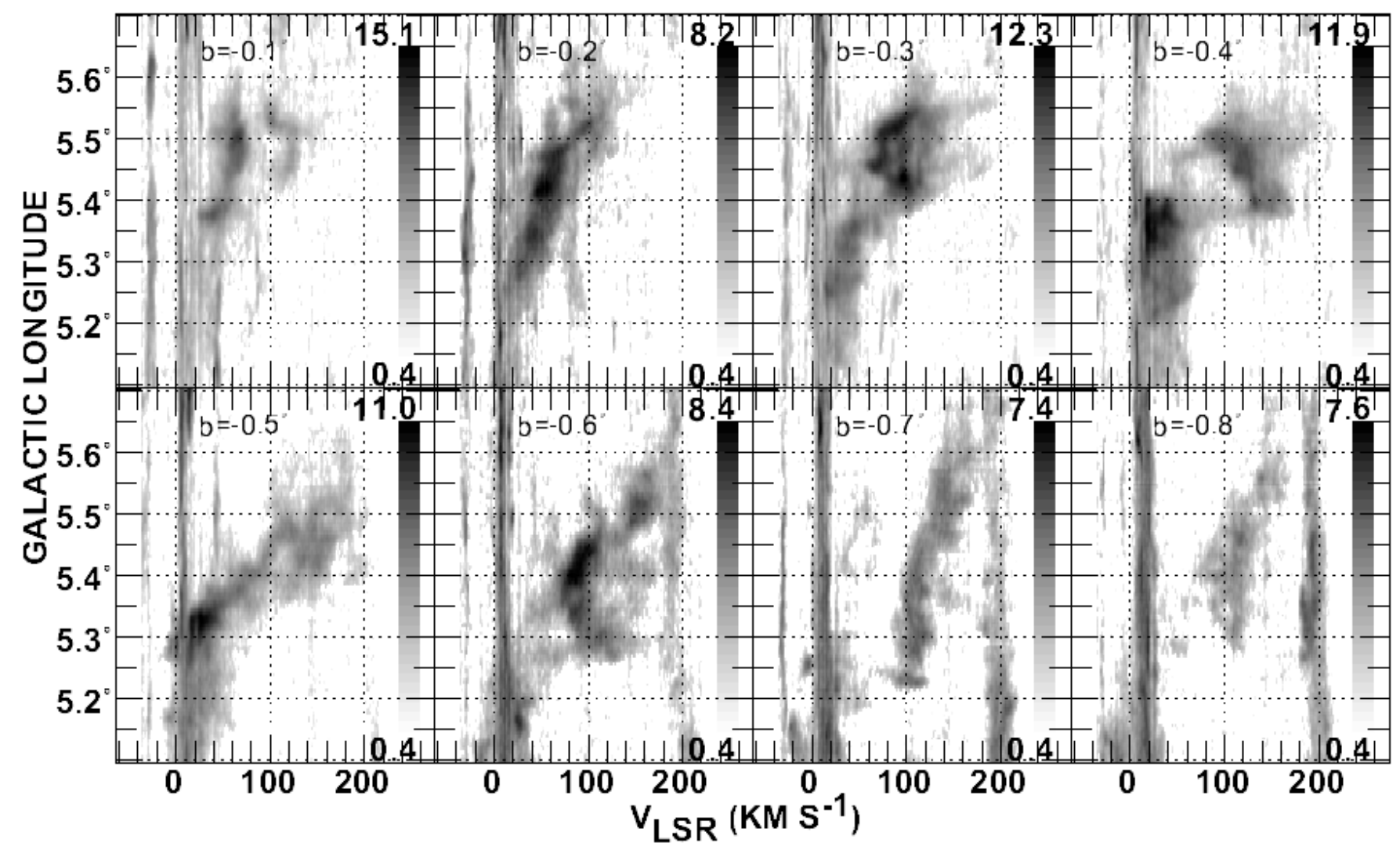

Fig. 6. Longitude-velocity cuts of ${ }^{12} \mathrm{CO}$ emission across the feature at $l=5.4^{\circ}$, spaced $0.1^{\circ}$ in latitude. The wide line of Fig. 2 at $b=-0.5^{\circ}$ is resolved into gas with a constant velocity gradient over $0.2^{\circ}$ or about $30 \mathrm{pc}$.

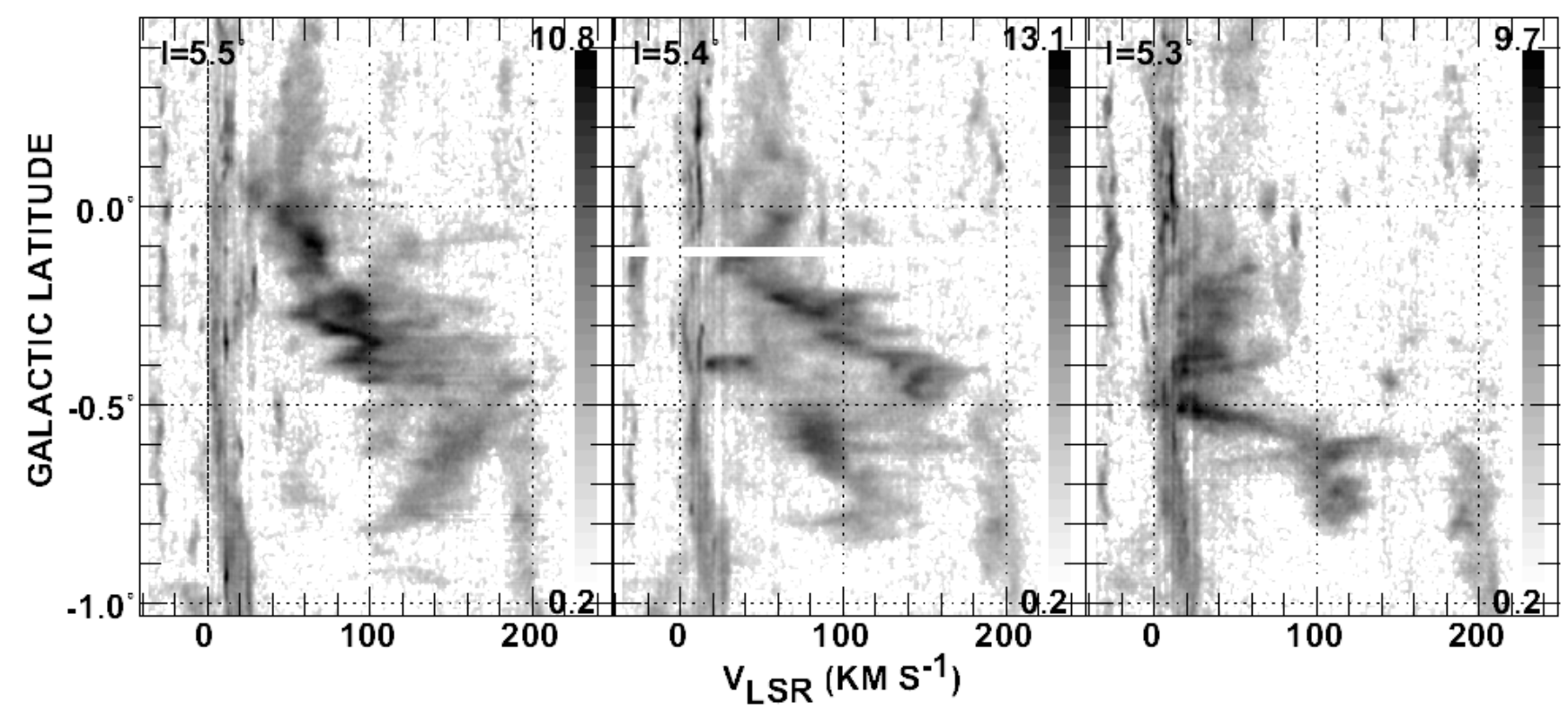

Fig. 7. Latitude-velocity cuts of ${ }^{12} \mathrm{CO}$ emission along the vertical length of the feature at $l=5.4^{\circ}$, spaced $0.1^{\circ}$ in longitude. The wide line of Fig. 2 at $b=-0.5^{\circ}$ is resolved into a large velocity gradient across $0.2^{\circ}$ in $l$. Systematic gradients and contrary, energetic, chaotic behaviour are clearly visible.

negative-latitude portions of Clump 2 were neglected in the earlier data.

Figure 9 shows a latitude-velocity slice through the center of the feature at $l=1.3^{\circ}$, and Fig. 10 is taken at $l=359.5^{\circ}$, both in the ${ }^{12} \mathrm{CO}$ line.

\subsection{The feature at $I=5.4^{\circ}$}

The integrated intensity map is given in the left-most panel of Fig. 4; the overall shape is narrow-waisted, centrallybrightened and slightly hooked, extending up and out of the 


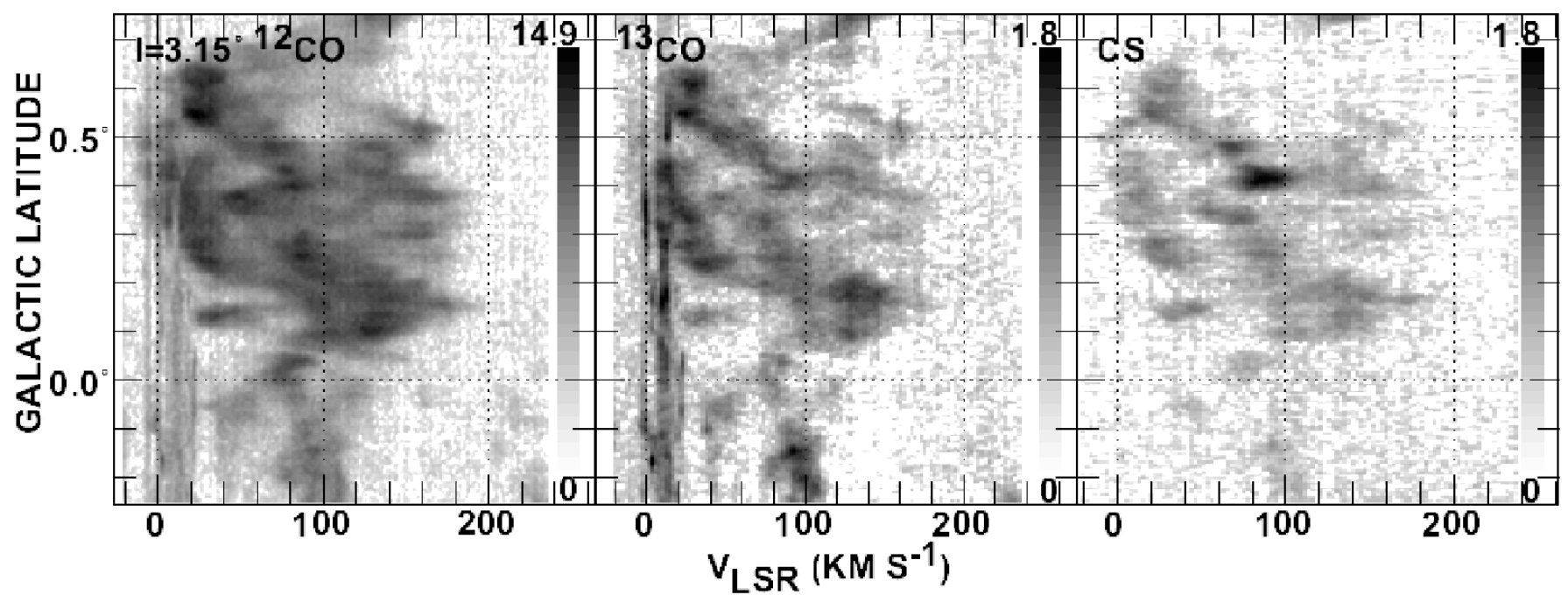

Fig. 8. Latitude-velocity cuts for ${ }^{12} \mathrm{CO},{ }^{13} \mathrm{CO}$ and $\mathrm{CS}$ as in Fig. 4 , but for the feature at $l=3.2^{\circ}$ aka Clump 2 of Stark \& Bania (1986). Note the marked resemblance to structure in Figs. 4 and 7.

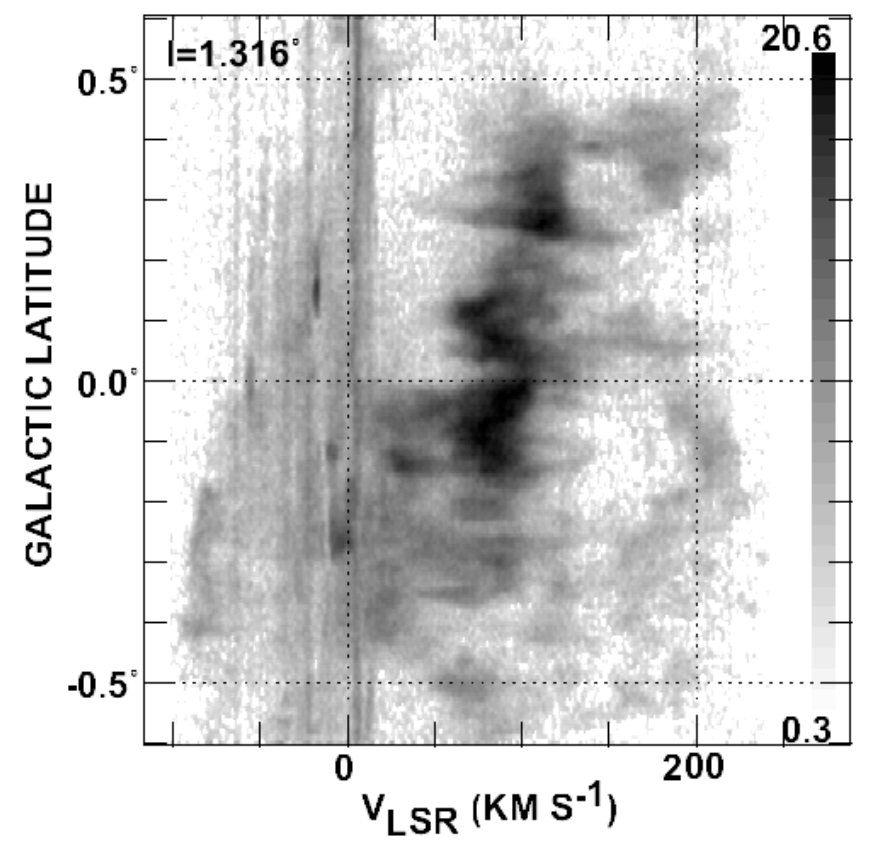

Fig. 9. A latitude-velocity slice at $l=1.32^{\circ}$.

tilted plane of the terminal-velocity ridge. As shown in the accompanying latitude-velocity slices, the inherent ${ }^{12} \mathrm{CO}$ line profiles are broad, but not as extensive as the larger-scale differences in velocity from top to bottom. The large-scale velocity structure at this longitude is fairly simple, mainly a decrease in velocity with latitude, and, clearly, very well-ordered. On small scales, however, there are very sharp localized gradients and velocity reversals (see also Fig. 7). Over its length, the feature occupies essentially the entire range of velocity between $20 \mathrm{~km} \mathrm{~s}^{-1}$ and the terminal velocity. Brighter (in ${ }^{12} \mathrm{CO}$ ) and denser (CS) material occupies (only) the central regions both in space and velocity. The gas in question is part of a welldefined body or phenomenon and does not arise from the superposition of unrelated molecular clouds. Consistent with the middle panel of Fig. 4 , the terminal velocity $\left(v \gtrsim 185 \mathrm{~km} \mathrm{~s}^{-1}\right)$ is actually occupied only at $b \lesssim-0.4^{\circ}$ and only in ${ }^{12} \mathrm{CO}$.

Figure 5 shows the spatial structure of the gas over narrow velocity ranges $\left(15 \mathrm{~km} \mathrm{~s}^{-1}\right)$. At $30-80 \mathrm{~km} \mathrm{~s}^{-1}$ the panels give the impression of a fountaining of gas nearer to and above the galactic equator, as multiple branches or arms leave the central trunk of emission in clear arcs oriented away from the largescale curvature of the feature as a whole. It is an interesting but somewhat tedious exercise to trace the velocity gradients seen so clearly in Figs. 6 and 7 in these moment maps.

Figure 6 shows the longitudinal velocity gradients observed over a wide range of latitude. The velocity gradient across the structure is generally well-resolved at arcminute $(2.5 \mathrm{pc})$ resolution (but note the abrupt behaviour in the panel at $b=-0.4^{\circ}$ ) and is largest around $b=-0.5^{\circ}$ where the gas is not particularly dense. Although there are individually broad profiles, the wide lines which appear so remarkable in the $8^{\prime}$ resolution data of Bitran et al. (1997) arise from the beam-smearing of velocity structure. The panels of this figure illustrate the overall North-South velocity gradient. Although there is some contrary behaviour, the overall gradient in longitude is one of velocity increasing with galactic longitude. Confinement of the gas motions below the terminal velocity is especially clear in the panels at lower latitude.

Figure 7 illustrates that the same phenomenon may be repeated internally; note the two parallel strands seen at $l=5.4^{\circ}$ (and the marked resemblance to behaviour in the middle panel of Fig. 8). But none of the features having large vertical velocity gradients in the panels of Fig. 7 exactly overlap. The rightmost panel shows the remarkable kinematic intersection of two $>100 \mathrm{~km} \mathrm{~s}^{-1}$-wide features with oppositely-directed velocity gradients, again illustrating the finding that the largest velocity gradients are vertical. Taken together Figs. 6 and 7 provide insight into how such a vertically extended feature stands out in the low-resolution data as a broad line seen over a relatively small range of galactic latitude. 


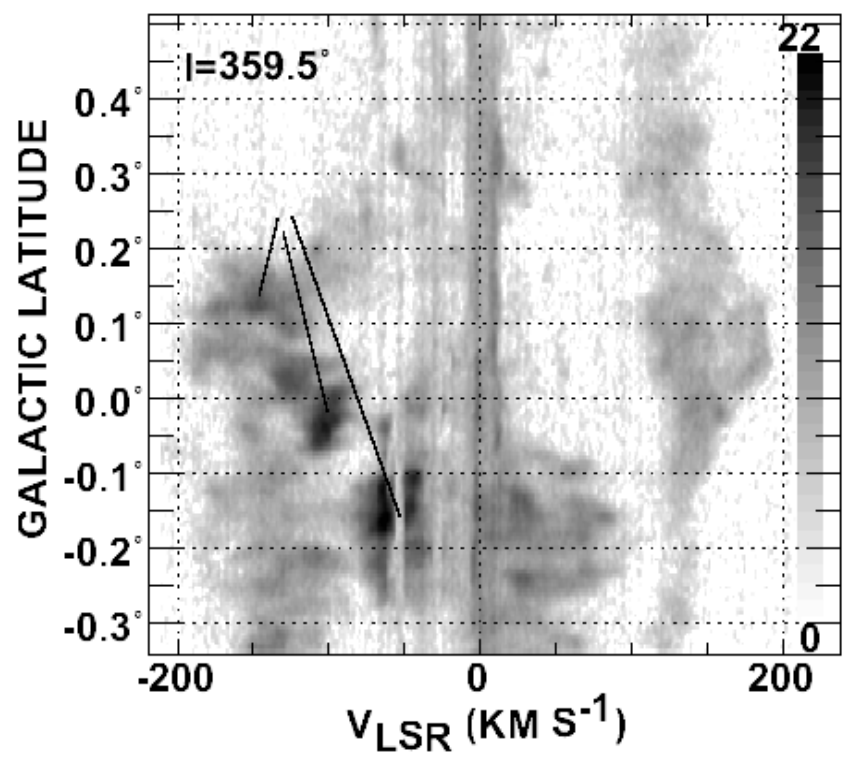

Fig. 10. A latitude-velocity slice at $l=359.5^{\circ}$. Brighter-lined gas particularly associated with the vertical structure at this longitude is marked. The Sgr C molecular cloud is associated with the bright emission at $-50 \mathrm{~km} \mathrm{~s}^{-1}$ around $b=-0.15^{\circ}$.

\subsection{Clump 2, the feature at $I=3.2^{\circ}$}

Latitude velocity cuts through the core of Clump 2 are shown in Fig. 8: unfortunately, our map does not go to quite high enough latitude to have captured all of the gas. However, the observed kinematics bear a truly remarkable resemblance to those seen at $l=5.4^{\circ}$ in the middle panel of Fig. 7 and would seem to leave little doubt that the same phenomena are seen in both features. Clump 2 is very slightly brighter. Physical conditions in Clump 2 and the feature at $l=5.4^{\circ}$ are discussed in Sect. 4 and the kinematics in Sect. 5.2.

\subsection{The feature at $I=1.3^{\circ}$}

A vertical cut through the broad line feature at $1<l<2^{\circ}$ is shown in Fig. 9. This is the only feature which appears as a broad line in both of the widely-separated panels of Fig. 2 but it has not previously been identified as participating in the phenomena described here. It is the only feature mapped here whose mid-point appears so symmetrically disposed about the galactic equator. Although the brighter-lined trunk of the feature is quite nearly vertical as it crosses the galactic plane, there is a noticeable curvature below the plane with a radius similar to that seen for the features at higher longitude (see Fig. 2). Emission from this feature comprises part of the pattern employed by Binney et al. (1991) to illustrate the $x_{2}-x_{1}$ orbit separation.

This is the second-brightest (in $\mathrm{CO}$ ) broad line feature and the second-closest to the center. It is marked by a very gradual gradient $\mathrm{dv} / \mathrm{db}$ with a sign opposite to those at higher longitude, but with the same jagged internal kinematics - abrupt velocity shifts and highly localized, occasionally contrary and perhaps

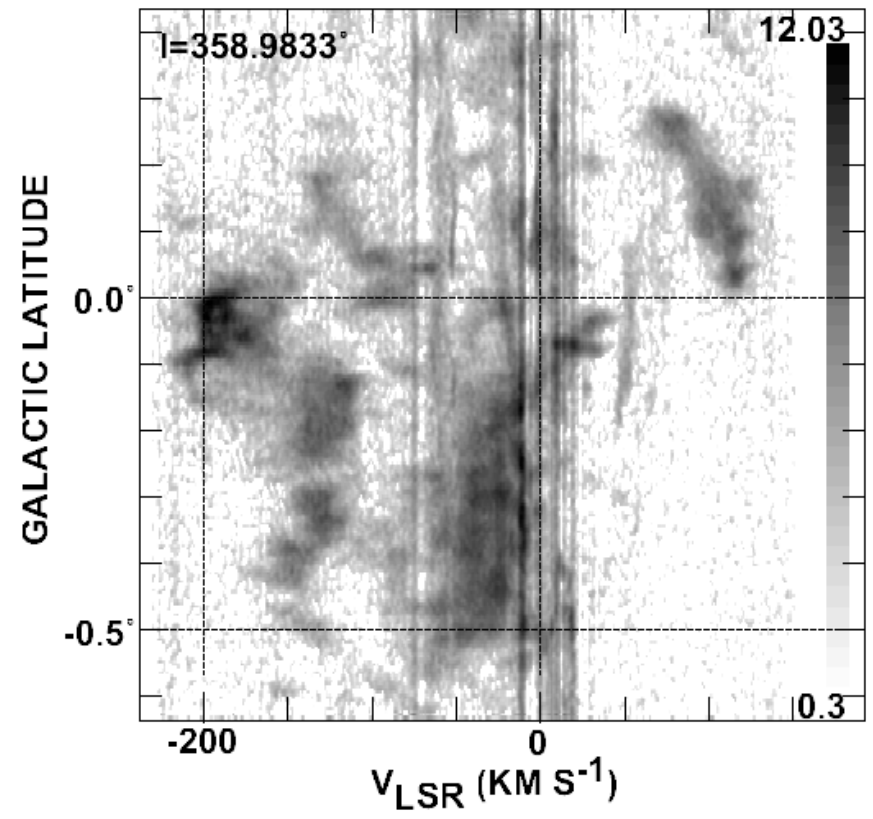

Fig. 11. A latitude-velocity slice at $l=358.98^{\circ}$. Gas particularly associated with the vertical structure seen at this longitude in Fig. 3 occurs at $v<0 \mathrm{~km} \mathrm{~s}^{-1}$.

even superposed velocity gradients of opposite sign - extending between 0 -velocity and the $200 \mathrm{~km} \mathrm{~s}^{-1}$ terminal velocity in patches of as much as $100-150 \mathrm{~km} \mathrm{~s}^{-1}$.

\subsection{The feature at $I=359.5^{\circ}$}

Figure 10 shows a feature with a large velocity gradient $d v / d b$ nested within other emission at the longitude of Sgr C (Liszt 1985; Liszt \& Spiker 1995). In fact, the Sgr C molecular cloud is the strongly emitting gas at $-50 \mathrm{~km} \mathrm{~s}^{-1}$ which is absorbed by the colder material in the $3 \mathrm{kpc}$ arm (ibid). This feature is usually considered to be the closest to the center and has the highest peak brightness seen in ${ }^{12} \mathrm{CO}(22 \mathrm{~K})$, despite being partly absorbed by foreground material.

\subsection{The feature at $I=359^{\circ}$}

Figure 11 shows a latitude velocity cut through the center of the vertically-extended feature which appears in Fig. 3 at $l=359^{\circ}$. In the absence of spatial mapping, the kinematics remain somewhat vague. The widely-extended gas below the galactic plane generally appears in two separate velocity intervals at $v<100 \mathrm{~km} \mathrm{~s}^{-1}$ and $v>100 \mathrm{~km} \mathrm{~s}^{-1}$. Emission at $b \sim 0^{\circ}$, $v \sim-200 \mathrm{~km} \mathrm{~s}^{-1}$ and $b \gtrsim 0^{\circ}, v \gtrsim 100 \mathrm{~km} \mathrm{~s}^{-1}$ was used by Liszt $\&$ Burton (1978) to illustrate the front-back tilt of the innerGalaxy gas distribution. The continuum sources in Sgr E (Liszt 1992) are seen at only slightly lower longitude, in association with the gas at $-210 \mathrm{~km} \mathrm{~s}^{-1}$. The Sgr E sources probably mark the outer boundary of the nuclear ring of star formation in the Galaxy, in the South. 


\subsection{Other wide lines}

There are wide lines in Fig. 2 which do not appear clearly in Fig. 3 and were not mapped here. The most striking example is Clump 1 of Bania (1977) at $l=-4.2^{\circ}$. Moreover, Fig. 2 shows only a small fraction of the data. Given the nature of the explanation expounded here for the presence of the broad lines, ubiquity is neither unexpected nor problematic: they could and perhaps should appear liberally over the galactic center region. Of more interest and lacking an explanation is why the most major examples typically are so heavily structured in the vertical direction (along which motions are largely undetectable, except for the tilted geometry of the inner galaxy gas).

\section{Physical conditions, masses, densities}

We drew a hull around the figure of the integrated CO brightness for the feature at $l=5.4^{\circ}$ in Fig. 4 and summed over the enclosed points. The area comprised some 28000 pixels (of size $15^{\prime \prime}$ or $0.6181 \mathrm{pc}$ ) with characteristic physical dimensions of $180 \times 60 \mathrm{pc}$ (their product defining the enclosed area). The enclosed integrated $\mathrm{CO}$ brightness was $5 \times 10^{6} \mathrm{~K} \mathrm{~km} \mathrm{~s}^{-1}$, for a mean of $180 \mathrm{~K} \mathrm{~km} \mathrm{~s}^{-1}$ per pixel.

For a typical galactic conversion from $\mathrm{CO}$ to molecular hydrogen $\left(2 \times 10^{20} \mathrm{H}_{2} \mathrm{~cm}^{-2}\right)\left(\mathrm{K} \mathrm{km} \mathrm{s}^{-1}\right)^{-1}$ the total mass would be $6.1 \times 10^{6} M_{0}$ and the mean column density would be $3.6 \times 10^{22} \mathrm{H}_{2} \mathrm{~cm}^{-2}$. Taking a $100 \mathrm{pc}$ dimension as characteristic of the depth, the mean density is inferred to be of order $100 \mathrm{H}_{2} \mathrm{~cm}^{-3}$. Repeating this exercise over the region of the bright southerly cores at $-0.3<b<-0.6^{\circ}$, we found a mass of $2.5 \times 10^{6} M_{0}$ (half the total) over a region of size $70 \times 45 \mathrm{pc}$, or a mean density of $400 \mathrm{H}_{2} \mathrm{~cm}^{-3}$.

The mass of the feature at $l=5.4^{\circ}$ derived in this way is nearly equal to that found for Clump 2 by Stark \& Bania (1986) who attributed their value to the presence of 16 dense, $\left(2 \times 10^{4} \mathrm{H}_{2} \mathrm{~cm}^{-3}\right)$ unrelated and unbound cores of typical mass $5 \times 10^{5} M_{0}$, having a small filling factor. Similar core and total mass estimates were presented by Boyce et al. (1989), based on the locally-derived abundances of $\mathrm{H}_{2} \mathrm{CO}$ and $\mathrm{OH}$. Apparently, the wide variety of locally-based mass estimates are quite consistent. But more importantly, the mean densities are both moderate and consistent with the limited extent of CS emission. Although the argument is usually made that the $\mathrm{CO}-\mathrm{H}_{2}$ conversion factor is smaller in the galactic center than locally, the features at $l=3.2^{\circ}$ and $l=5.4^{\circ}$ are seen 500-800 pc away. Stark \& Bania (1986) justified their mass estimates by calculating the stability of the cores against galactic shear and similar arguments concerning the observed linewidths were expressed by Boyce et al. (1989). In our interepretation (see Sect. 5), gas within the broad-line features is actually in the process of being shocked and shredded and the stability argument is probably moot.

The overall ${ }^{12} \mathrm{CO} /{ }^{13} \mathrm{CO}$ line brightness ratio in the widelined features is much larger than is typically seen in the galactic plane outside the center, but the ${ }^{12} \mathrm{CO} / \mathrm{CS}$ ratio is actually not. Over the extent of the latitude-velocity cuts in Figs. 3 and 8 where we have comparable data in all three lines, the ratio of total observed brightnesses (in the sense ${ }^{12} \mathrm{CO}:{ }^{13} \mathrm{CO}: \mathrm{CS}$ ) is remarkably similar in the two regions, $1: 0.055: 0.025$ at $l=5.4^{\circ}$ and 1:0.056:0.027 at $l=3.15^{\circ}$. Observations of species beside ${ }^{12} \mathrm{CO}$ in the galactic plane show that the ${ }^{12} \mathrm{CO} /{ }^{13} \mathrm{CO}$ brightness ratio is typically 4-5 while species having high dipole moments typically have brightnesses of order $2 \%$ that of ${ }^{12} \mathrm{CO}$, as seen here (Liszt 1995; Helfer \& Blitz 1997).

As indicated by Fig. 4, detectable ${ }^{12} \mathrm{CO}$ emission is more extensive than that of either ${ }^{13} \mathrm{CO}$ or $\mathrm{CS}$, in space and velocity. We find that, for the feature at $l=5.4^{\circ}$, comparison between profiles of the three species is very much as shown by Stark \& Bania (1986). That is, ${ }^{13} \mathrm{CO}$ or CS covers the same range as ${ }^{12} \mathrm{CO}$ but very non- uniformly; within the line profile, there are regions having very high and much smaller ${ }^{12} \mathrm{CO} / \mathrm{CS}$ or ${ }^{12} \mathrm{CO} /{ }^{13} \mathrm{CO}$ brightness ratio (within the limits imposed by sensitivity, ${ }^{13} \mathrm{CO}$ and $\mathrm{CS}$ are very similar). At $l=5.4^{\circ}$ denser gas occupies mostly the region of the dense Southerly core. However, for the feature at $l=3.2^{\circ}$, this is actually not the case: the panels in Fig. 8 are much more nearly scaled replicas of each other, both parallel strands in the central panel of Fig. 8 show strong CS (compare the central panel in Fig. 4) etc. For the feature at $l=3.2^{\circ}$ the more typical comparison (on a point by point basis, since spatial averaging will enforce conformity) is that all three species have not only similar support in velocity, but similar line shape as seems clear from Fig. 8. At $l=3.2^{\circ}$ dense gas seems more uniformly spread (detectable?) over the region, whatever its filling factor.

To summarize, the overall ${ }^{12} \mathrm{CO} /{ }^{13} \mathrm{CO}$ brightness ratio in either feature is relatively large compared to observations in the galactic plane, perhaps characteristic of warm gas having moderate density or optical depth. The masses derived for the features at $l>3^{\circ}\left(5 \times 10^{6} M_{0}\right)$ in a variety of ways are comparable to those of the largest GMC's seen outside the galactic center and do not imply high density. They would be substantial even if, ad hoc, they were reduced by a factor 5 .

\section{What are the broad-lined features, really?}

\subsection{Alignment of the bar and Sun-center line}

The phenomena noted here can only be interpreted in the context of a particular viewing geometry. With respect to Fig. 1, the two hypothetical viewing angles will yield galactic center kinematics with some common but mostly disparate essential elements. Both will show a strong pattern of circular motion over the inner region from gas in the nearly-round and mostlyrotating nuclear ring. However, the bar side-on view will show only small non-circular motion toward the center. This view exposes the dust-lane shock over a wider range of longitude, and post-shock gas in the dust lane, moving mostly across the line of sight (in the frame of the bar at least) will appear at smaller $|v|$; the terminal velocity ridge (largest $|v|$ ) is composed of preshock gas moving nearly along the line of sight. For the more nearly end-on view, the circular component of the nuclear ring will still be present, but the strong non-circular motion in the spray will be seen toward the center, dust-lane post-shock gas moves more nearly along the line of sight, forming the terminal velocity ridge, and it is the preshock gas which appears at velocities between zero and that of the terminal velocity ridge. 


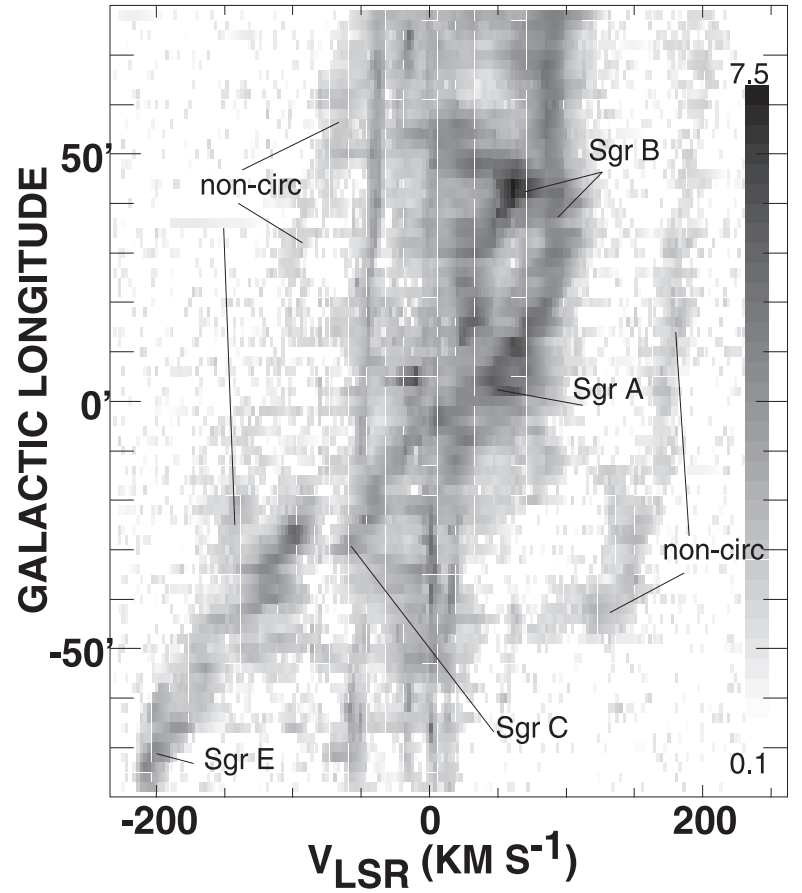

Fig. 12. Rotation pattern of stronglined- ${ }^{13} \mathrm{CO}$ emission illustrating the predominantly circular motion of the gas harboring the HII regions in the central region, and the presence of gas components dominated by circular and non-circular motion along the same line of sight.

Figure 12 shows a longitude-velocity diagram of ${ }^{13} \mathrm{CO}$ emission at the galactic latitude of $\mathrm{Sgr} \mathrm{A}^{*}$ upon which are marked some well-known distinguishing characteristics. The well-separated galactic center giant $\mathrm{H}$ II regions Sgr A, B, C, and E (not D) show a strong rotation signature (Liszt et al. 1977; Liszt 1992) within a more continuous pattern of strong-lined $\mathrm{CO}$ emission which we identify with the nuclear ring in Fig. 1: the speed of this pattern is $210 \mathrm{~km} \mathrm{~s}^{-1}$ and the radius is $70^{\prime} \approx 175 \mathrm{pc}$. Although other material may exist at smaller radii, the structure is ring-like (as opposed to, say, a disk) because high rotation speeds are only seen well away from the center. The lack of kinematic symmetry in the ring at higher positive longitudes - the absence of gas at $v>100 \mathrm{~km} \mathrm{~s}^{-1}$ in the rotation signature at $l \gtrsim 1^{\circ}-$ can be cured by observing above the galactic equator (Burton \& Liszt 1992). Although the galactic center HII regions all appear at very nearly the same galactic latitude, the front and back sides of the nuclear ring are distinguishable in both space and velocity because the ring gas is slightly tilted with respect to the galactic equator and because the motion within the gas is, systematically, slightly non-circular. This will be discussed in more detail in a forthcoming paper.

Clearly, strong non-circular motions are present in Fig. 12 at positive velocity and the absence of stronger forbidden velocity emission at $v<0$ is really due only to the tilt of the gas (see the next subsection): this is the well-known "expanding molecular ring" (EMR) gas, which in the current context is not a material body but the manifestation of spray at the inner ends of the dust lane shocks ${ }^{1}$. There can be no doubt that the viewing geometry is not side-on: indeed, the more nearly end-on viewer in Fig. 1 is about at the extreme of the range of angle (between the bar major axis and Sun-center line) previously inferred. In this case, we have that post-shock gas in the dust lane at larger longitudes moves more nearly along the line of sight than does the pre-shock gas, and so will appear at larger $|v|$, forming the terminal velocity ridge. As noted above, this ridge is generally very clear in $\mathrm{CO}$ and absent in those molecular tracers which probe higher-density gas.

\subsection{The tilt(s) of the galactic center gas}

As shown here, the overall tilt of the large-scale gas distribution plays a substantial role in the observed gas kinematics of the broad-lined features. The feature at $l=5.4^{\circ}$ appears to emanate or terminate well below the galactic plane, where the tilt seen across the line of sight has carried the terminal velocity ridge. Put differently, the gas layer and/or dust lane appears to slope down in the first quadrant of longitude and up in the fourth. Another aspect of the tilt is partly responsible for the weakness of the EMR gas in Fig. 12 at negative velocity. Negative-velocity $\left(-135 \mathrm{~km} \mathrm{~s}^{-1}\right)$ EMR gas appears more prominently at latitudes below that of Sgr A while the positivevelocity portion appears $\left(+165 \mathrm{~km} \mathrm{~s}^{-1}\right)$ at higher latitudes (see Fig. 10 of Liszt \& Burton 1978).

Assuming that the inner-galaxy gas layer has a mid-plane, the orientation of this mid-plane requires specification of two angles in space. In the original models made by this author, both angles were used to explain the dust-lane tilt and latitude separation of the positive and negative velocity portions of the EMR gas seen toward Sgr A. However, with regard to Fig. 1, if it is imagined that the view is more nearly end-on, and if the near end of the bar slopes down below the galactic equator (which only requires one angle), the near and far portions of the spray (seen as the EMR) will also separate in latitude in the correct sense of yielding negative velocities at lower galactic latitude around Sgr A. Thus in a bar model like that in Fig. 1, these two seemingly independent aspects of the tilt are really quite similar in origin. This does, however, leave the specification of the second angle somewhat vague.

The second angle required to specify the complete spatial orientation of the putative gas mid-plane can be used either to moderate or strengthen the apparent slope $\mathrm{d} b / \mathrm{d} l$ of the gas. If the gas cants downward from right to left on the sky - if the upward normal appears in the first quadrant of galactic longitude - this could sharpen some of the effects; gas at a constant $z$-height above the mid-plane would appear at even higher latitude at smaller longitude, which may have been observed for the feature at $l=3.2^{\circ}$, as discussed below.

\subsection{Dynamical sequence at $I=5.4^{\circ}$}

According to the preceding discussion, post-shock gas in the features at positive longitude is in the terminal velocity ridge at

\footnotetext{
${ }^{1}$ Ironically, there is a ring in both the model and the Milky Way, but it is not the "expanding" feature.
} 
higher $v$, while pre-shock gas appears at smaller velocity. Thus, contrary to the possible impression of upward spray in Fig. 5, the kinematic ordering is from smaller to larger velocity and the spatial sequence of the overall gas processing is, generally, both downward and from lower to higher longitude. Gas nearer $b=0^{\circ}$, initially at smaller velocity and longitude, is being gathered and condensed, brightened in CO and especially in CS and other high-density tracers before entering the dust lane and moving on into the center in, apparently, a lower-density and more uniform medium. That is, the terminal velocity ridge is ubiquitous in carbon monoxide and $\mathrm{H} \mathrm{I}$ but generally absent in other, molecular tracers of higher-density gas.

Somewhat contrary behaviour is also seen. The higherdensity gas at $b \leq 0.5^{\circ}, v=120-180 \mathrm{~km} \mathrm{~s}^{-1}$ in Fig. 4 or in the end panels of Fig. 7 has a contrary slope dv/db. It seems hardly coincidental that this behaviour appears to join with the terminal velocity ridge just where the other gas should be entering the dust lane shock. This might be gas reflected from the shock, gas entering the shock from below, or perhaps gas affected in some other way, due to the strong local self-gravity.

\subsection{Other features, other mysteries}

The similar kinematic patterns observed in Figs. 4 and 8 for the features at $l=5.4^{\circ}$ and $l=3.2^{\circ}$ seem to demand similar physical effects. Yet, it is harder to make a consistent narrative at $l=3.2^{\circ}$ because the terminal velocity ridge and putative dust lane/shock appear well below $b=0^{\circ}$ even at this longitude and do not really appear in Fig. 8 at all. Furthermore, the terminal velocity ridge, where it $i s$ seen at lower latitudes at $l=3.2^{\circ}$ is at $v=230-250 \mathrm{~km} \mathrm{~s}^{-1}$, to which the motions in Fig. 8 do not extend. The pre-shock material, if it is indeed the gas at lower velocity, is at rather high latitude. Finding gas at higher galactic latitude and smaller longitude is, however, consistent with the sense of the one obvious tilt and could be sharpened by specification of both tilt angles, as mentioned in Sect. 5.2. Our data on this feature cover only a small part of it; perhaps a more detailed examination of the older data of Stark \& Bania (1986) is in order.

Our maps of the other features are too incomplete to provide such a detailed narrative as was possible for the feature at $l=5.4^{\circ}$. At $l=1.3^{\circ}$ in Fig. 9 we see the larger vertical extent of strong $\mathrm{CO}$ emission, jagged internal kinematics and some locally very broad line profiles; according to Fig. 3 this feature extends below $b=-1^{\circ}$, well beyond the extent of the strip we mapped.

Given the ubiquity of shocks in the structure associated with gas flow in the bar, it seems likely that lesser examples of broad lines - perhaps not quite so broad, or so extended will be scattered over the galactic center region.

\section{Summary}

We decomposed the inner-galaxy molecular gas distribution into two parts following a clear morphological distinction between gas in the terminal velocity ridge - which is in a relatively confined plane and tilted at about $22^{\circ}$ with respect to the galactic equator - and that seen at smaller velocity. Within the latter we identified a family of some six remarkable features in the galactic center neutral gas distribution, two of which have previously been noted for their properties observed in broad beams. The distinguishing observational characteristics of this family are:

- Highly localized, seemingly isolated, strong, wide $\left(200 \mathrm{~km} \mathrm{~s}^{-1}\right)$ lines in broad $\left(\sim 8^{\prime}=20 \mathrm{pc}\right)$ beams, which resolve spatially to large velocity gradients along the galactic plane (typically, $200 \mathrm{~km} \mathrm{~s}^{-1}$ over $0.2^{\circ}=30 \mathrm{pc}$ ) accounting, for the most part, for the widest lines seen in $l-v$ cuts made with larger beams at particular latitudes.

- In some cases, even larger gradients across the galactic plane and in general systematic and largely monotonic change of velocity with latitude.

- Occasional very broad profiles even in regions extending only a few minutes of arc (3-10 pc) and jagged internal kinematic structure with both contiguous and overlapping large, sometimes contrary, velocity gradients.

- Great elongation across the galactic equator and large vertical extent (up to $1^{\circ}=150 \mathrm{pc}$ or more). The features are generally slightly curved at one or both ends.

- Vertical structure which appears to recognize the large- scale tilts of the inner-galaxy gas, in particular the terminal velocity ridge which is identified with the bar dust lanes.

- Relatively bright ${ }^{12} \mathrm{CO}$ lines $(12 \mathrm{~K}-20 \mathrm{~K}$ or more) and density enhancements (CS emission) at intermediate velocities, but large overall ${ }^{12} \mathrm{CO} /{ }^{13} \mathrm{CO}$ brightness ratios (15) typical of warm, not very dense gas, and overall $\mathrm{CO} / \mathrm{CS}$ ratios (40) like those seen in the galactic plane. Features seen closer to the center are systematically brighter in ${ }^{12} \mathrm{CO}$.

- High masses but moderate mean densities, typically $5 \times$ $10^{6} M_{0}$ and $100 \mathrm{H}_{2} \mathrm{~cm}^{-3}$ for locally-based mass estimates from $\mathrm{CO}, \mathrm{CS}, \mathrm{H}_{2} \mathrm{CO}, \mathrm{OH}$ etc. in the two cases mapped here in the most detail.

We attributed the presence of these features to material traversing standing shocks at the inside edges of the Milky Way's inner-galaxy dust lane, which in hydrodynamical models of gas flow in strong bars naturally accounts for the widespread occurence of such localized, broad lines. To interpret the sequence of events associated with the kinematic organization within the major features most completely mapped here, we discussed the viewing geometry of the galactic bar from the Sun. We established detailed correspondences between the strongest kinematic signatures in the galactic center gas distribution with particular features expected of gas flow in strong fast bars. That is, we associated the presence of a strong rotation signature in the motion of the Sagittarius H II regions with a nuclear star forming ring of radius $175 \mathrm{pc}$, rotating at $210 \mathrm{~km} \mathrm{~s}^{-1}$. The strong non-circular motions observed in $\mathrm{H} \mathrm{I}$ and molecular gas toward Sgr A $\left(-135 \mathrm{~km} \mathrm{~s}^{-1},+165 \mathrm{~km} \mathrm{~s}^{-1}\right)$ are explained by "spray" of inflowing gas at the inner ends of the dust lanes, necessitating (as has typically been found in previous discussions) a situation in which the bar major axis is seen more nearly end on than side on. 
With this orientation, the latitude separation of the positive and negative velocity portions of the expanding molecular ring gas (in the sense that velocity and sign of latitude are the same around Sgr A) is naturally explained by the same tilt angle which so prominently carries the dust lane to negative latitude at positive longitude. Also with this orientation it follows that the terminal velocity ridge at highest $|v|$ in our line profiles arises from gas inflowing along the dust lanes. We noted that the terminal velocity ridge is visible only in $\mathrm{H} \mathrm{I}$ and $\mathrm{CO}$, and not in molecular tracers of higher density gas (which strikingly exhibit the broad- lined features).

Much of the most important structure of the gas, both on large scales seen across the galactic center and within individual broad-lined features, is vertical; the strongest overall radial-velocity gradients within the latter are in galactic latitude and (Fig. 3) the feature at $l=5.4^{\circ}$ is strikingly oriented such that its lower end, nearly 100 below the galactic plane, intersects the highly inclined dust lane/terminal velocity ridge. Given the orientation of the bar, we showed how, for the feature at $l=5.4^{\circ}$, gas is actually falling down from the region of the galactic equator, being gatherered and condensed (brightened in $\mathrm{CO}$ and $\mathrm{CS}$ ) at intermediate velocities and negative latitudes, before being sucked into the dust lane $100 \mathrm{pc}$ below the galactic equator. This gas is presumably being channeled (and, because of the tilt, lifted) back into the galactic center nearer the galactic equator, seemingly sustaining the overall organization.

Of course, the vertical structure and tilt of the inner-galaxy gas remain something of a mystery and vertical motions themselves are unobservable unless they are normal to the tilted gas plane: the phenomena seen here remain to be explained in detail. A forthcoming paper will discuss the kinematics of gas in the Milky Way's nuclear star-forming ring, which itself has interesting warping and vertical structure. The importance of such rings for evolution of galactic nuclei has recently been stressed by Regan \& Teuben (2003) and Regan \& Teuben (2004).

Acknowledgements. The NRAO is operated by AUI, Inc. under a cooperative agreement with the US National Science Foundation. I thank Drs. T. Dame and P. Thaddeus for providing the datacube of Bitran et al. (1997). Thanks to Steward Observatory for having the faith in the $12 \mathrm{~m}$ telescope which the NSF and NRAO lacked. Thanks to W. Butler Burton for insight and encouragement and to Mike Regan for enlightening discussions on barred-spiral gas dynamics.

\section{References}

Athanassoula, E. 1992, MNRAS, 259, 328

Bania, T. M. 1977, ApJ, 216, 381

Bania, T. M., Stark, A. A., \& Heiligman, G. M. 1986, ApJ, 307, 350

Binney, J., Gerhard, O. E., Stark, A. A., Bally, J., \& Uchida, K. I. 1991, MNRAS, 252, 210

Bitran, M., Alvarez, H., Bronfman, L., May, J., \& Thaddeus, P. 1997, ApJS, 125, 99

Boyce, P. J., Cohen, R. J., \& Dent, W. R. F. 1989, MNRAS, 239, 1013

Burton, W. B., \& Liszt, H. S. 1978, ApJ, 225, 815

Burton, W. B., \& Liszt, H. S. 1983, A\&AS, 52, 63

Burton, W. B., \& Liszt, H. S. 1992, ApJS, 95, 9

Cohen, R. J., \& Davies, R. D. 1976, MNRAS, 175, 1

Contopoulos, G., \& Grosbol, P. 1989, Astr. Ap. Rev., 1, 261

Fux, R. 1999, A\&A, 345, 787

Gerhard, O. E. 1996, in Unsolved Problems of the Milky Way, IAU Symp., 169, 79

Helfer, T. T., \& Blitz, L. 1997, ApJ, 478, 233

Jenkins, A., \& Binney, J. 1994, MNRAS, 270, 703

Kumar, P., \& Riffert, H. 1997, MNRAS, 292, 871

Lake, G., \& Norman, C. 1983, ApJ, 270, 51

Lee, C. W. 1996, ApJS, 105, 129

Lee, C. W., Lee, H. M., Ann, H. B., \& Kwon, K. H. 1999, ApJ, 513, 242

Liszt, H. S. 1985, ApJ, 293, L65

Liszt, H. S. 1992, ApJS, 82, 495

Liszt, H. S. 1995, ApJ, 442, 163

Liszt, H. S., \& Burton, W. B. 1978, ApJ, 226, 790

Liszt, H. S., \& Burton, W. B. 1980, ApJ, 236, 779

Liszt, H. S., Burton, W. B., Sanders, R. H., \& Scoville, N. Z. 1977, ApJ, 213, 38

Liszt, H. S., \& Spiker, R. W. 1995, ApJS, 98, 259

Mulder, W. A., \& Liem, B. T. 1986, A\&A, 157, 148

Oort, J. H. 1977, ARA\&A, 15, 295

Peters, W. L. 1975, ApJ, 195, 617

Regan, M. W., Sheth, K., \& Vogel, S. N. 1999, ApJ, 526, 97

Regan, M. W., \& Teuben, P. 2003, ApJ, 599, 999

Regan, M. W., \& Teuben, P. J. 2004, ApJ, 600, 595

Rohlfs, K. 1983, A\&A, 128, 426

Rohlfs, K., \& Braunsfurth, E. 1982, A\&A, 113, 237

Sawada, T., Hasegawa, T., Handa, T., et al. 2001, ApJS, 136, 189

Stark, A. A., \& Bania, T. M. 1986, ApJ, 306, L17

Stark, A. A., Martin, C. L., Walsh, W. M., et al. 2004, ApJ, 614, L41

Thaddeus, P. 1985, Bull. Am. Astron. Soc., 17, 606

Vietri, M. 1986, ApJ, 306, 48

Weiner, B. J., \& Sellwood, J. A. 1999, ApJ, 524, 112

Zylka, R., Güsten, R., Henkel, C., \& Batrla, W. 1992, A\&AS, 96, 525 